\title{
Multiview ISAR Imaging for Complex Targets Based on Improved SBR Scattering Model
}

\author{
Tiantian Feng $\mathbb{D}$ and Lixin Guo \\ School of Physics and Optoelectronic Engineering, Xidian University, Xi'an 710071, China \\ Correspondence should be addressed to Lixin Guo; lxguo@xidian.edu.cn
}

Received 16 November 2020; Revised 16 December 2020; Accepted 13 January 2021; Published 25 January 2021

Academic Editor: Ping Li

Copyright (c) 2021 Tiantian Feng and Lixin Guo. This is an open access article distributed under the Creative Commons Attribution License, which permits unrestricted use, distribution, and reproduction in any medium, provided the original work is properly cited.

\begin{abstract}
A novel multiview inverse synthetic aperture radar (ISAR) imaging method is proposed to simulate high-resolution and identifiable ISAR image of complex targets by handling large-angle and wide-bandwidth scattering data. The scattering data are simulated with the shooting and bouncing ray (SBR) method. The bidirectional ray-tracing algorithm is developed to reduce the computation time. Simulation results indicate that the improved method is efficient and reliable to calculate electromagnetic (EM) scattering of electrically large targets. To implement the multiview ISAR imaging method after data simulation, we divide the large-angle and wide-bandwidth scattering data into subaperture data and conduct ISAR image processing for each subaperture datum locally. Transforming each subaperture ISAR image to the global coordinate system and summing them together will produce the high-resolution ISAR image that is meaningful for the database set up for synthetic aperture radar automatic target recognition (SAR ATR). The final simulation ISAR images further validate the great performance of our scattering calculation algorithm and ISAR imaging method.
\end{abstract}

\section{Introduction}

Inverse synthetic aperture radar (ISAR) imaging has been widely used in target detection and recognition due to its operation capacity of all day and all weather [1]. Many methods such as bandwidth extrapolation $[2,3]$ and compressive sensing (CS) $[4,5]$ have been studied in past several years to obtain high-resolution ISAR images. These methods focus on how to generate more radar data with the parameterization processing algorithm from the aspect of signal processing. Imaging with electromagnetic scattering (EM) echoes data simulated by EM scattering algorithms is another method to achieve ISAR imaging. This method can provide theoretical foundation for ISAR imaging and is significant to ISAR imaging experiment. EM scattering simulation algorithms are divided into low-frequency numerical method and high-frequency approximation method. The former method includes method of moments (MOM) $[6,7]$ and multilevel fast multipole method (MLFMM) [8]. The computational ability of these methods is limited by the target size. The shooting and bouncing ray (SBR) method [9-12] is a high-frequency method which combines the physical optics (PO) method and geometrical optics (GO) method. It can calculate the far field scattering of electrically large targets efficiently. In the SBR method, the intersection tests are quite time-consuming especially as the target size increasing. Many acceleration techniques, such as ray-box intersection algorithm in FPGA [13, 14], multiresolution grid algorithm [15], recursive subdivision octree algorithm [16], and KD-tree based algorithm [17], have been developed to reduce the computation time of ray-tracing process. In this report, the bidirectional ray-tracing algorithm is proposed to decrease the intersection tests between patches and rays, further reducing computation time.

Automatic target recognition (ATR) of synthetic aperture radar (SAR) has been significantly applied in military tasks [18]. The SAR ATR system requires a large ISAR image database of numerous computer-aided design (CAD) targets for all observation angles to compare with test targets to be classified and recognized [18, 19]. The multiview ISAR 
imaging method is quite essential to create the database of CAD targets for various observation angles. In [20-23], methods for multistatic ISAR imaging of moving targets have been presented. The study [20] describes the ISAR processing and fusion by coherent/incoherent summation in different overlapping situations of the radar network. The authors in $[21,22]$ develop the 3D reconstruction method based on a multistatic InISAR system. In recent years, circular synthetic aperture radar (SAR) [24] is developed as a novel SAR mode. It is achieved by steering the radar platform to rotate in a circular trajectory. The imaging process includes generating subimages and the followed incoherent procedure. Inspired by this circular trajectory concept, we made attempt to conduct the multiview ISAR imaging simulation according to the subaperture principle $[25,26]$. In the proposed multiview ISAR imaging method, the backward scattering data of a target are obtained from multiple observation locations and stored as multiple sets of subaperture data. Synthesizing these subaperture data is equivalent to increasing the total imaging aperture; thus, the image resolution will be greatly improved. In this technique, each subaperture datum is simulated by the introduced SBR method in large-angle range and wide bandwidth.

The rest of this paper is organized as follows. In Section 2 , the principle of the bidirectional ray-tracing algorithm is described and followed by backward scattering simulations of a dihedral reflector as well as two electrically large targets. The comparison of simulation results between MLFMM and the improved SBR method is given to validate the correctness of our method. Then, the theoretical model of the multiview ISAR imaging method using large-angle widebandwidth subaperture data is figured out. Section 3 shows numerical examples including the comparison of image quality of large-angle and wide-bandwidth ISAR imaging between the direct FFT method and the PRA method and multiview ISAR imaging simulations of the missile and airplane model. The results of the multiview ISAR imaging simulation confirm the validity of our method.

\section{Theoretical Model}

2.1. Backward Scattering Simulation by SBR Method. In the SBR method, the target model is subdivided into small triangle patches and the incident wave is assumed as dense grids of ray tubes shot toward the target. The propagation direction of the incident ray can be determined according to the law of GO. The backscattered field of illuminated patches will be calculated by PO. Although the SBR method is more effective compared to numerical algorithms, the ray-tracing process for electrically large target is still time-consuming. Thus, we proposed the bidirectional ray-tracing algorithm to reduce the computation time during ray-tracing process.

As shown in Figure 1, the incident ray reflected by patch $A$ of the plate 1 intersects patch $B$ of the plate 2. First, we can search neighbor patches of patch $B$ (that is, patch C, patch D, and patch $\mathrm{E}$ ) according to $\mathrm{KD}$-tree of the target model. Then, assume that the rays are shot from the neighbor patches in the direction reverse to that of the first reflected ray from patch A. If the reverse direction rays intersect with patch A,

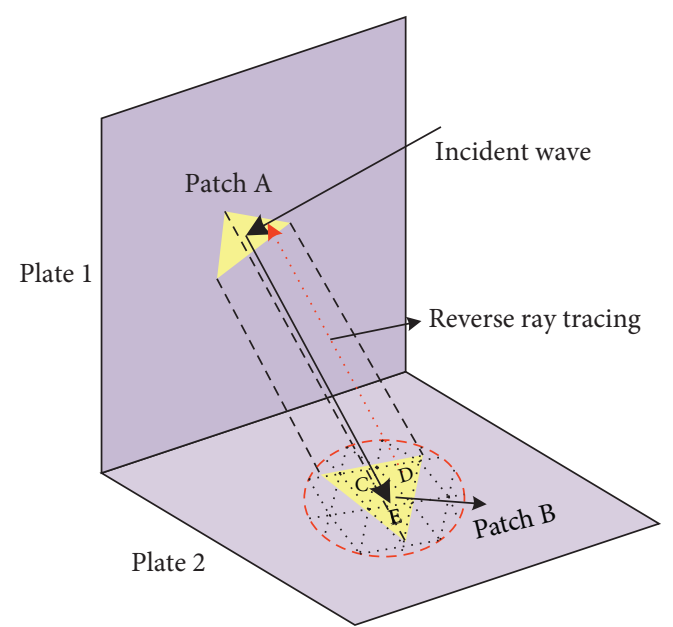

Figure 1: Illustration of bidirectional ray tracing.

then their original patches are illuminated by the first reflected field from patch A. Subsequently, the same reverse ray-tracing process will be conducted on the illuminated neighbor patches one by one. As for the computation about the second reflection, we replace the patches illuminated by the incident wave by the ones illuminated by first reflected rays. Finally, far field scattering of illuminated triangles found out by the ray-tracing process should be calculated, respectively, by physical optics (PO) as follows:

$$
\vec{E}^{s}=\frac{j k \exp (-j k R)}{4 \pi R} \iint \eta \cdot \widehat{k}_{s} \times\left(\widehat{k}_{s} \times \vec{J}\right) \exp j k \widehat{k}_{s} \cdot \vec{r}^{\prime} d s^{\prime},
$$

in which $\eta=\left(\varepsilon_{0} / \mu_{0}\right)^{1 / 2} ; \vec{J}$ is the current density at $\vec{r}^{\prime}$ on the triangle patch.

Figure 2 shows monostatic RCS of a dihedral reflector at $10 \mathrm{GHz}$. The side length of the dihedral reflector is $0.3 \mathrm{~m}$. The results of our method are compared with those of MLFMM in FEKO. It can be seen that the two sets of data match well. However, the simulation results of the PO method present significant difference, which indicates the effect of multiple reflections on the RCS simulation of such dihedral construct. In the following, we applied the SBR method to backward scattering data simulation of a missile. The length of the missile model is $6.8 \mathrm{~m}$, and the wingspan is $2.6 \mathrm{~m}$, as illustrated in Figure 3. The azimuthal angle of the incident wave changes from $0^{\circ}$ to $360^{\circ}$ at the elevation angle $\theta_{i}=30^{\circ}$. The backscattering RCS results at $10 \mathrm{GHz}$ for both $\mathrm{HH}$ polarization and VV-polarization are shown in Figure 4. It can be seen obviously that the RCS of the missile varies greatly with the incident angle and reaches to the peak value when the incident wave illuminates both vertical sides of the missile. To further verify the efficiency of the introduced SBR method, an airplane model with length $15 \mathrm{~m}$ and wingspan $9 \mathrm{~m}$ is set up (as shown in Figure 5). The monostatic RCS results for HH-polarization and VV-polarization are calculated as shown in Figure 6. The incident angles are set as $\varphi_{i}=0^{\circ} \sim 360^{\circ}, \theta_{i}=45^{\circ}$. As can be seen from the results, the RCS of the aircraft changes more significantly with the incident angle and gets the maximum value when the 


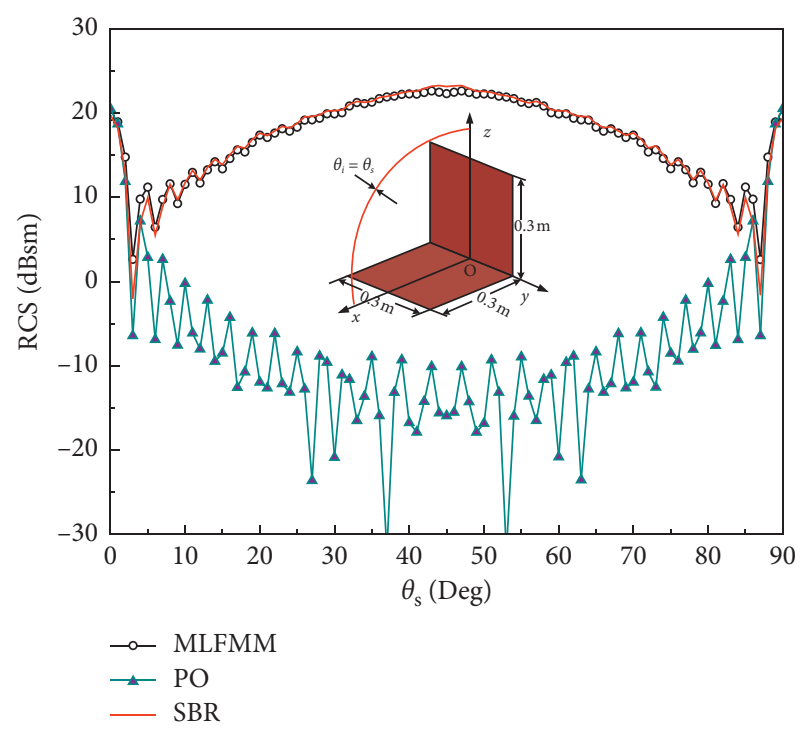

Figure 2: Monostatic RCS of dihedral reflector.

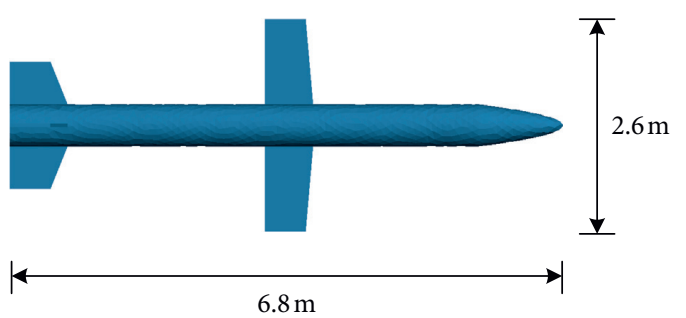

FIGURE 3: Geometry of the missile model.

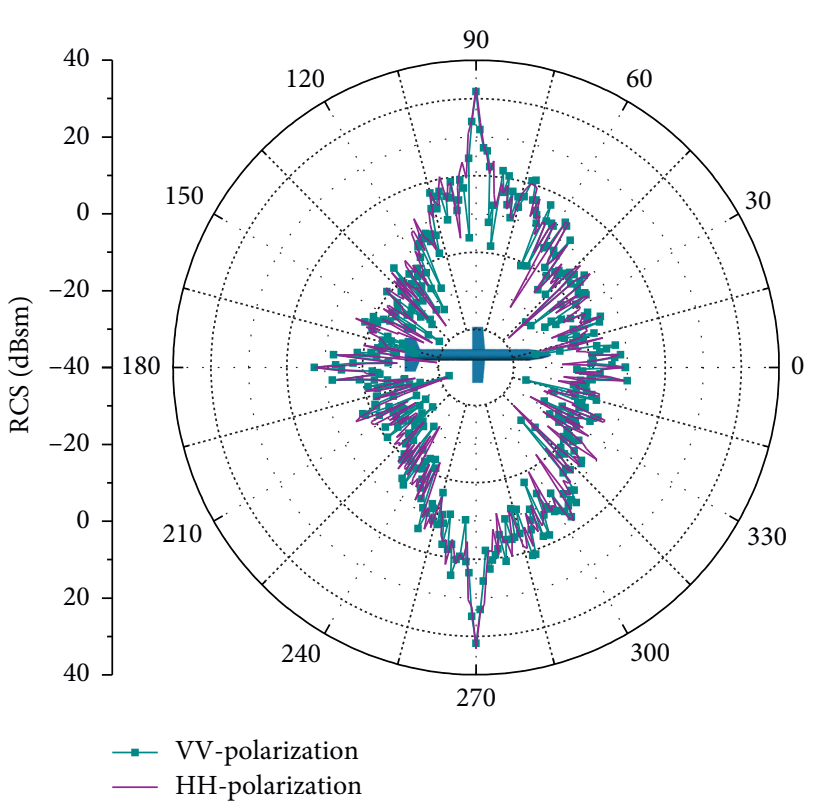

FIgURe 4: Monostatic RCS of a missile at $10 \mathrm{GHz}$.

incident azimuths are $90^{\circ}$ and $270^{\circ}$. Table 1 illustrates the comparison of runtime performance between the MLFMM in FEKO and the SBR method. It is worth to notice that the improved SBR method has achieved great speedup. All

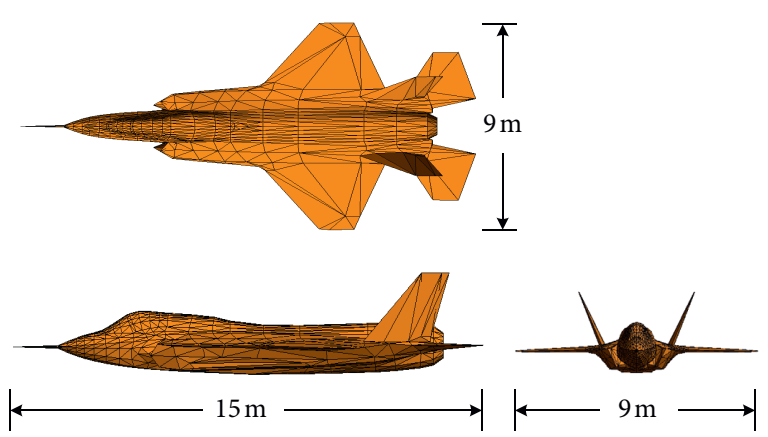

FIGURE 5: Geometry of the airplane model.

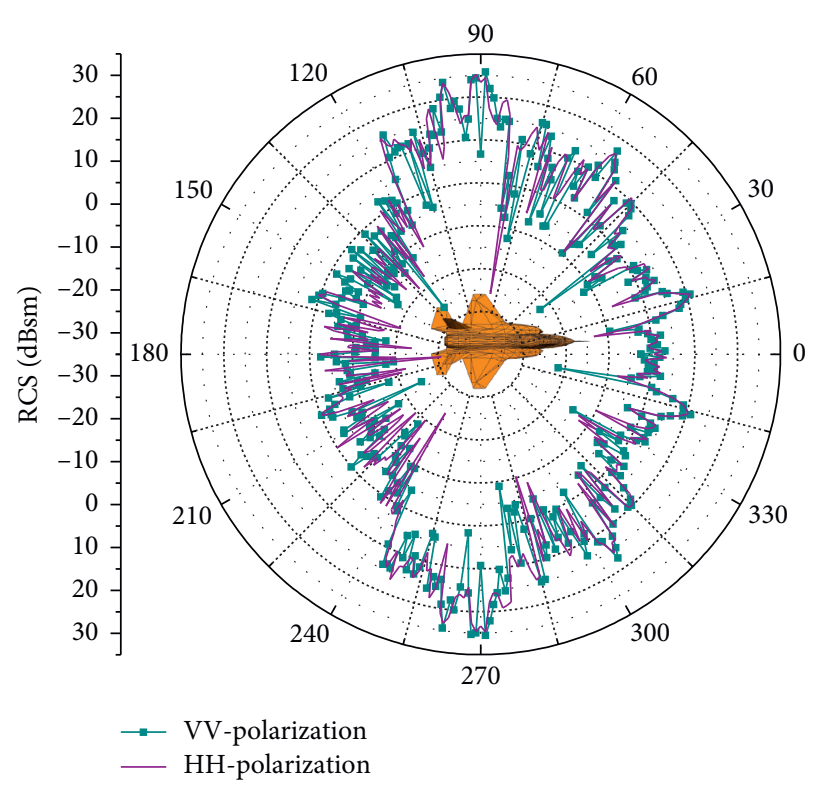

FIgURE 6: Monostatic RCS of airplane at $10 \mathrm{GHz}$.

simulation results indicate that the bidirectional ray-tracingbased SBR method is able to calculate the backscattering of complex targets with good accuracy and efficiency.

2.2. Multiview ISAR Imaging. The first two steps of the multiview ISAR imaging algorithm are collecting backscattering data from different looking angles (as shown in Figure 7) as subaperture data and obtaining subaperture ISAR images in their local coordinates. Each subaperture image is obtained using large-angle and wide-bandwidth subaperture data. Then, the subimages are transformed into the global coordinate system and synthesized to generate the total ISAR image.

The algorithm can be summarized as follows:

(i) Collect the backward scattering data for wide bandwidth and large angular range. Record the data as $\vec{E}(f, \varphi)$. In this report, the backward scattering data in the form of two-dimensional matrix can be obtained effectively by the above introduced SBR method for different looking angles at different frequencies. 
TABLE 1: Summary of runtime performance.

\begin{tabular}{lccc}
\hline Method & & MLFMM & SBR \\
\hline \multirow{3}{*}{ Runtime (s) } & Dihedral & 5197.3 & 4.2 \\
& Missile & - & 20.3 \\
& Airplane & - & 92.7 \\
\hline
\end{tabular}

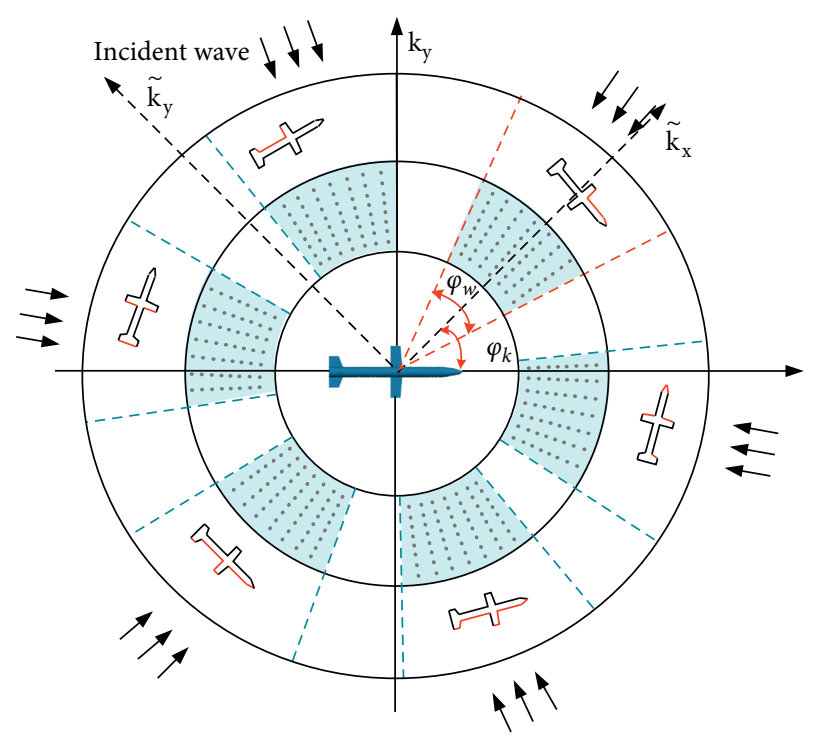

FIGURE 7: Illustration of multiview ISAR.

(ii) Repeat step (i) and obtain subaperture data from different observation locations for $N$ groups. These $N$ groups subaperture data are all in polar distribution.

(iii) Apply polar reformatting algorithm (PRA) and IFFT to obtain $N$ subaperture ISAR images utilizing $N$ group data from the former steps.

As is known to all, the ISAR image is proportional to the following integral:

$S(x, y) \sim \int_{\varphi_{1}}^{\varphi_{2}} \int_{k_{1}}^{k_{2}} \vec{E}(k, \varphi) \cdot \exp \left(j 2\left(k_{x} \cdot x+k_{y} \cdot y\right)\right) \mathrm{d} k \mathrm{~d} \varphi$,

in which

$$
\begin{aligned}
& k_{x}=k \cos \varphi, \\
& k_{y}=k \sin \varphi .
\end{aligned}
$$

Here, $\vec{E}(k, \varphi)$ is the backward scattering data of the target requiring ISAR image. If the backward scattering data are collected in large-angle range and wide bandwidth, it will be presented in the polar grid in the $k_{x}-k_{y}$ domain, as shown in the point array in Figure 7. In this situation, fast Fourier transform is inappropriate to be directly employed in imaging process. To transform the original polar format data into rectangular grid on the $k_{x}-k_{y}$ plane, $k_{x}$ and $k_{y}$ is resampled equally between the maximum and the minimum value, that is $[\min (k \cos \varphi), \max (k \cos \varphi)]$,

$[\min (k \sin \varphi), \max (k \sin \varphi)]$. The resampling process will generate a new $k_{x}-k_{y}$ grid. Then, every original scattering datum is set into the nearest point on the new $k_{x}-k_{y}$ grid. To minimize the error associated with this reformatting procedure, the four nearest neighbor scheme interpolation method is used in polar format data.

(iv) Transform each subimage from their local coordinate system into the global coordinate system by PRA introduced above.

Assuming there are $N$ subapertures, the center looking angle of each subaperture is $\varphi_{k}$ (as shown in Figure 7), where $k=1, \ldots, N$. The backscattered data of each subaperture are collected over azimuth angle $\varphi \in\left[\varphi_{k}-\phi_{w} / 2, \varphi_{k}+\phi_{w} / 2\right]$, where $\phi_{w}$ is the total scan azimuth angle of each subaperture. However, each subaperture has its own coordinate in the direction of $\varphi_{k}$. Let $\widetilde{\varphi}=\varphi-\varphi_{k}$ denote the local azimuth angle in the $k$ th subaperture's local coordinate system. $\widetilde{k}_{x}$ and $\widetilde{k}_{y}$ are the wave number in the $k$ th subaperture's local coordinate system. Then, the corresponding global coordinate wave number $k_{x}, k_{y}$ can be written as

$$
\begin{aligned}
& k_{x}=k \cos \left(\widetilde{\varphi}+\varphi_{k}\right)=\widetilde{k}_{x} \cos \varphi_{k}-\widetilde{k}_{y} \sin \varphi_{k}, \\
& k_{y}=k \sin \left(\widetilde{\varphi}+\varphi_{k}\right)=\widetilde{k}_{y} \cos \varphi_{k}+\widetilde{k}_{x} \sin \varphi_{k},
\end{aligned}
$$

where

$$
\begin{aligned}
& \widetilde{k}_{x}=k \cos \tilde{\varphi}, \\
& \tilde{k}_{y}=k \sin \widetilde{\varphi} .
\end{aligned}
$$

Form equations (4) and (5), we can see that the global coordinate image can be obtained from the local coordinate image by a rotation of $\varphi_{k}$. For the multiview ISAR image, the rotation angle is different for each subaperture.

(v) Sum up all the transformed subimages to get the total ISAR image of the target.

\section{Results and Discussion}

In this section, ISAR imaging for large angular bandwidth and wide frequency bandwidth using the direct FFT method and the PRA method is compared firstly. Afterward, the multiview ISAR imaging process is implemented on the realistic missile and airplane model, respectively. The subaperture backscattering data of missile model are presented in matrix form, and each subimage is illustrated then. Finally, the synthesized ISAR image generated by our method 
is compared with the small-angle and narrow-bandwidth ISAR image. All simulated ISAR images are in linear scale.

3.1. ISAR Imaging for Large-Angle Wide-Bandwidth Data. The top view of the missile model is shown in Figure 3. The length of the model is $6.8 \mathrm{~m}$, and the wingspan is $2.6 \mathrm{~m}$. During this simulation, the incident wave central frequency is set at $8 \mathrm{GHz}$. The bandwidth is $4 \mathrm{GHz}$ including 256 equally spaced frequency steps. Also, the azimuth angle was altered from $-15^{\circ}$ to $15^{\circ}$ with 256 sampling points.

Figure 8 shows the ISAR image obtained directly from IFFT. The image is blurred and unfocused because of the nonuniform distribution scattering data in the $k_{x}-k_{y}$ domain. After reformatting the data by the PRA, the image is shown in Figure 9. It can be seen that polar reformatting followed by IFFT processing provides very fine resolutions both on range and cross-range dimensions. Nevertheless, the numerical noise due to reformatting the data from polar format to the rectangular format is unavoidable.

3.2. Multiview ISAR Imaging for Missile Model. Next, the multiview ISAR image of the missile model is simulated. The incident wave frequency varies from $6 \mathrm{GHz}$ to $10 \mathrm{GHz}$ with 256 equally spaced frequency steps. We select 8 representative subapertures with center azimuth angle $0^{\circ}, 45^{\circ}, 90^{\circ}$, $135^{\circ}, \ldots, 315^{\circ}$, successively. The angle bandwidth of each subaperture is $30^{\circ}$ with 256 sampling points. The simulation steps are the following:

(1) Collect backscattered data over given angle and frequency bandwidth for each subaperture. This will provide a total of 8 different subaperture data, and each has $256 \times 256$ data value. The simulated backward scattering data in spatial-frequency domain are shown in Figure 10. It can be seen that in center azimuth $\varphi=90^{\circ}$ and $\varphi=270^{\circ}$, the backscattering data have obviously strong scattering points. This reflects the fact that the missile has strong backscattering when incident from vertical sides. In center azimuth $\varphi=0^{\circ}$ and $\varphi=180^{\circ}$, the backscattering is weaker but still has strong scattering part because of the warhead and wing structures. In the other center azimuths, the backscattering data vary with the frequency and look-angles. We can easily search the parameters for strong scattering, which provide important data support for the target detection and recognition of the SAR ATR system.

(2) Use the PRA method and IFFT to simulate the ISAR image of each subaperture. The backward scattering data processed with PRA in $k_{x}-k_{y}$ domain are shown in Figure 11. After polar reformatting, the data in Figure 10 have been presented in uniform rectangular in $k_{x}-k_{y}$ domain. Figure 12 illustrates eight sub-ISAR images in the eight different lookangles after IFFT. Each subimage is simulated in its

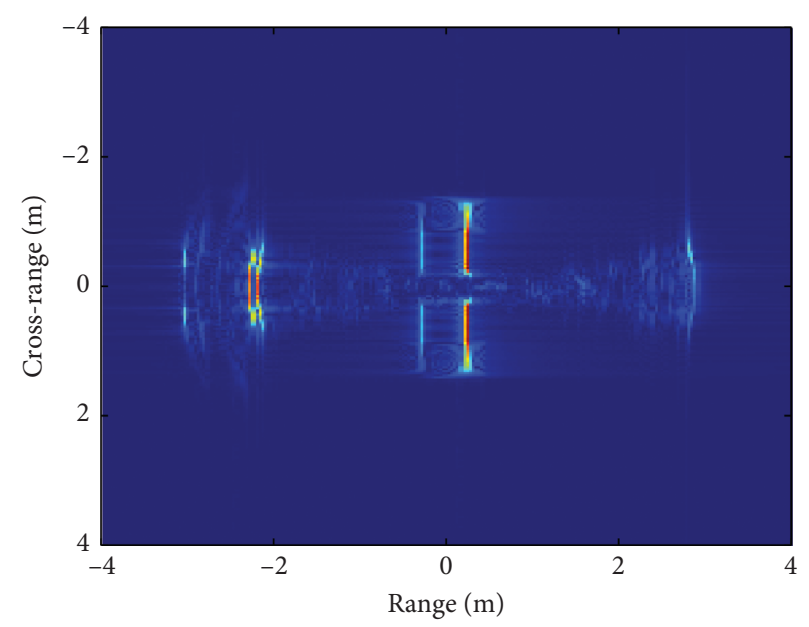

FIGURE 8: ISAR image with direct FFT for large-angle wide bandwidth.

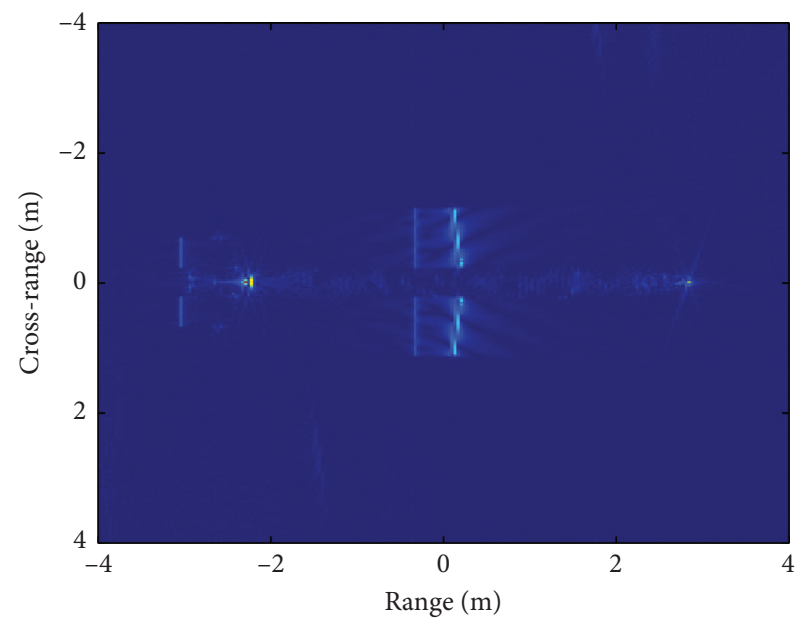

FIGURE 9: ISAR image with PRA for large-angle wide bandwidth.

local coordinate system. It can be seen that each subimage has high resolution and presents a part of the missile model. The two edges of missile are clearly outlined in 3th and 6th subimages when the center azimuth is $\varphi=90^{\circ}$ and $\varphi=270^{\circ}$, respectively. Meanwhile, the wings of the missile appear apparently in the 1th and 5th subimages. The 2th and the 8th subimages show the warhead of the missile, as the incident wave directly illuminates this part. As for the 4th and 6th subimages, the incident wave is oblique incident at the missile body, so the backscattering is not strong and the image is fuzzy.

(3) Rotate each subimage from the local coordinate system to the global coordinate system. The subimages in the global coordinate are shown in Figure 13.

(4) Finally, all subimages are summed up to form the final focused ISAR image, as shown in Figure 14(a). 

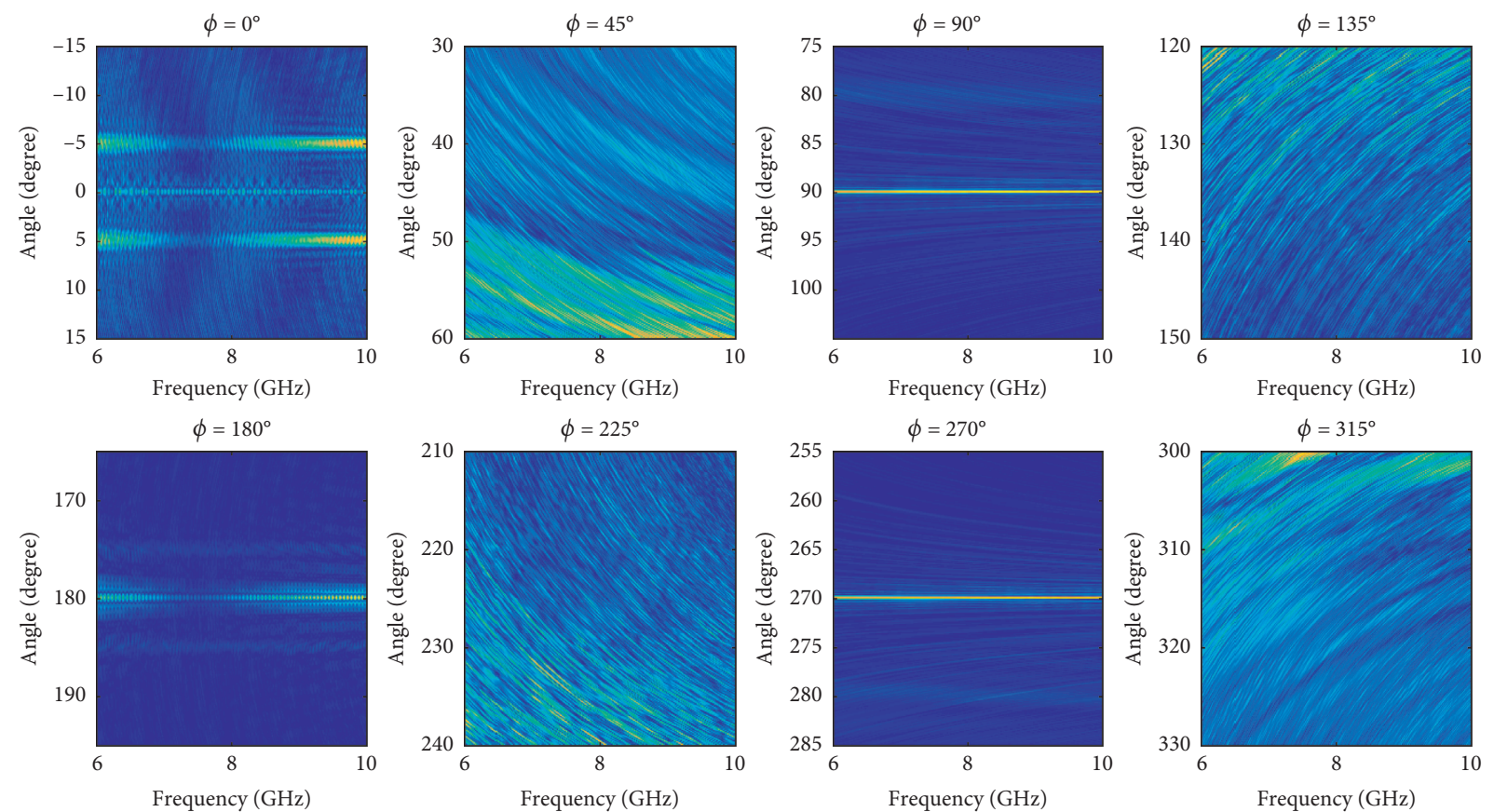

FIgURE 10: The backward scattering data in spatial-frequency domain.
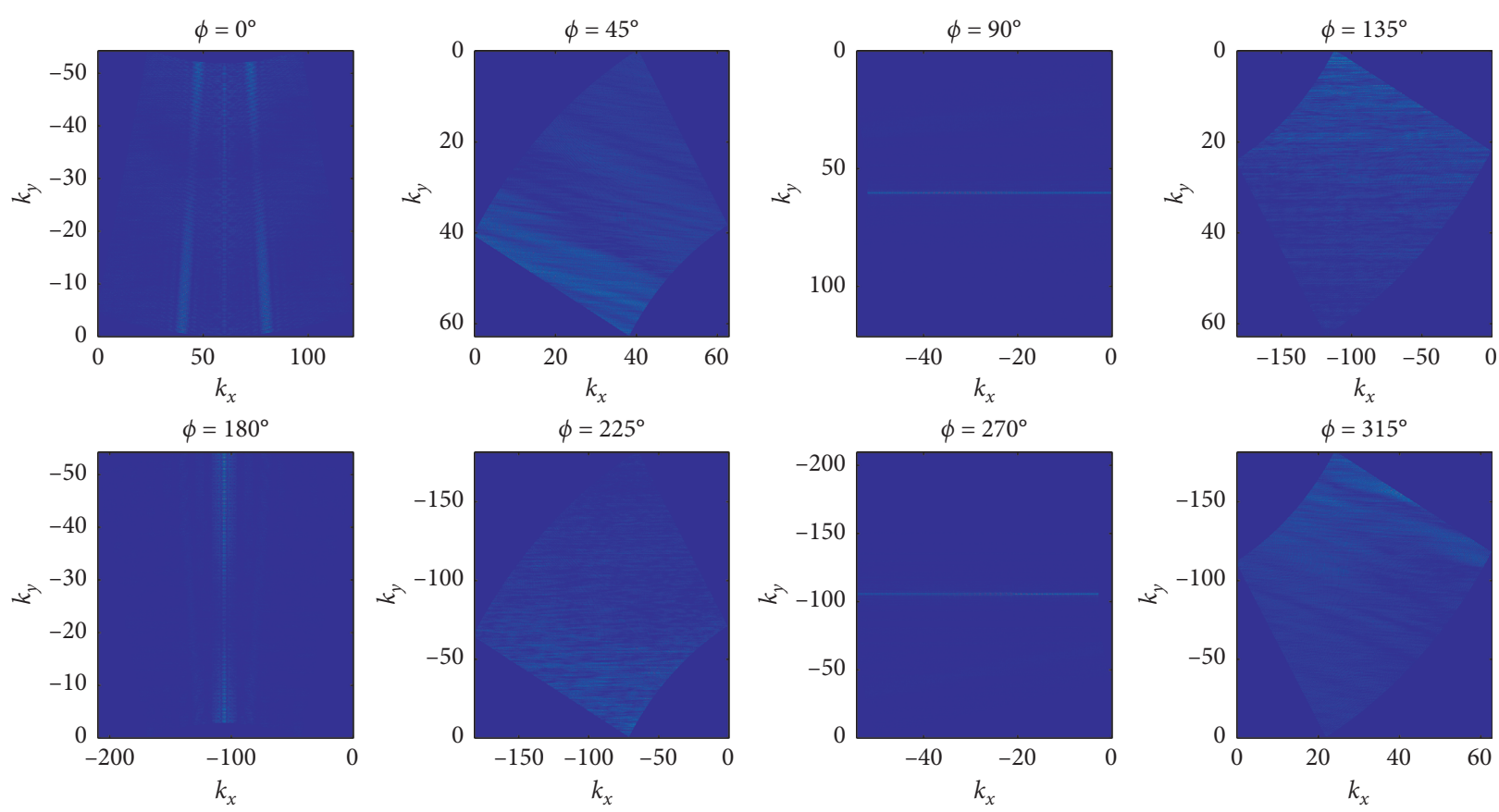

FIgURE 11: The backward scattering data in $k_{x}-k_{y}$ domain.

The final multiview ISAR image is well focused, and the missile is perfectly outlined. Figure 14(b) shows the multiview ISAR image simulated with small angle and narrow bandwidth data. The angle range is $8.6^{\circ}$ and the frequency bandwidth is $1.5 \mathrm{GHz}$ for each subaperture. We can see that the ISAR image in Figure 14(b) is unfocused and blurred compared with that in Figure 14(a). The left image better reflects the fine structure of the target.
3.3. Multiview ISAR Imaging for Airplane Model. In the following, an airplane model (the length is $15 \mathrm{~m}$, and the wingspan is $9 \mathrm{~m}$ ) is established as shown in Figure 5 . We select 8 same observation locations as those of the missile model. The central frequency is set as $6 \mathrm{GHz}$, and the bandwidth of each subaperture is $2 \mathrm{GHz}$. The azimuth range of each subaperture is fixed in $20^{\circ}$. Both frequency and azimuth sampling points are 256 . The multiview ISAR imaging procedure is the same as before. Figures 15 and 16 show the subimages of the 

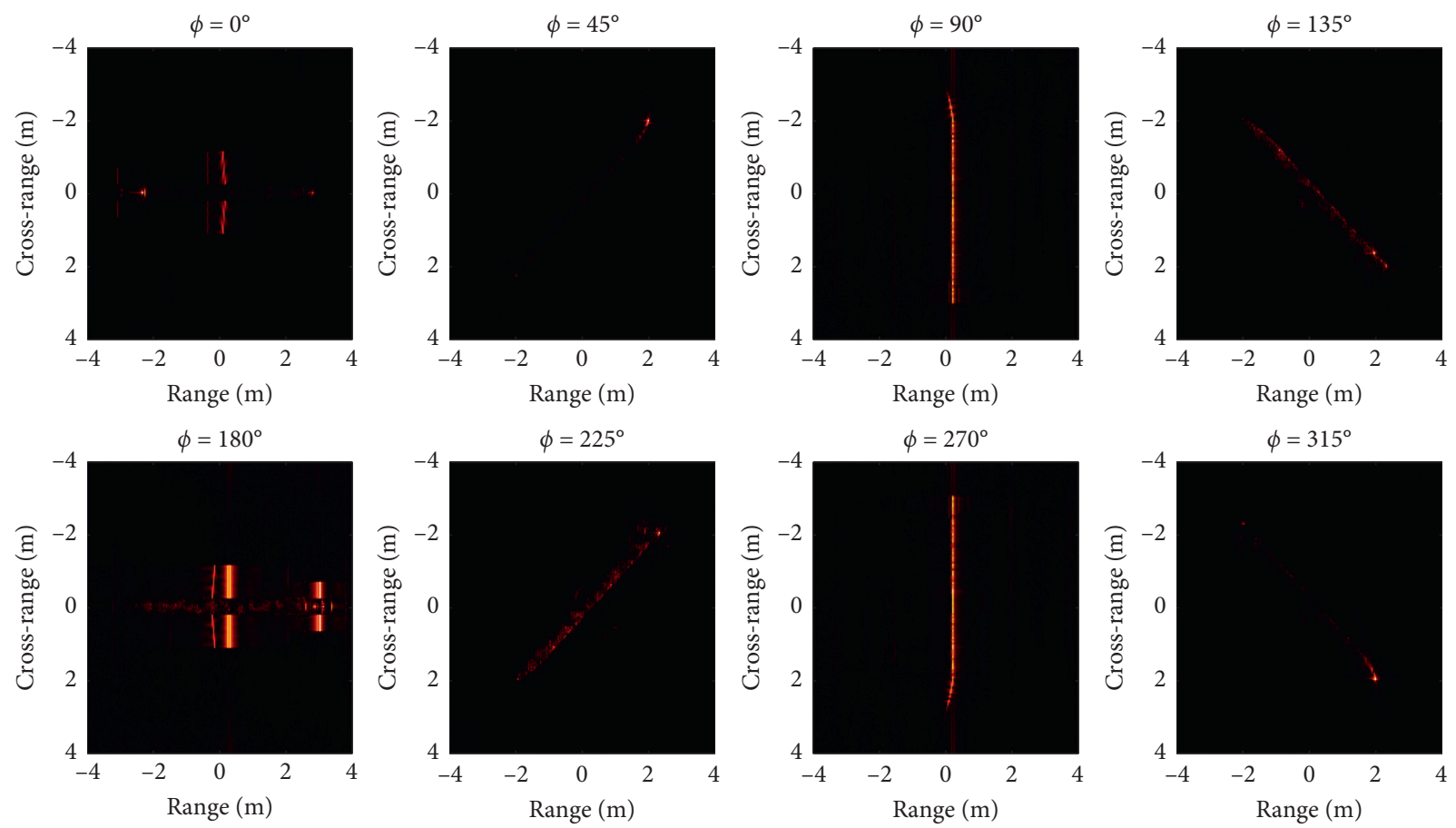

Figure 12: Subaperture images in different look-angles.
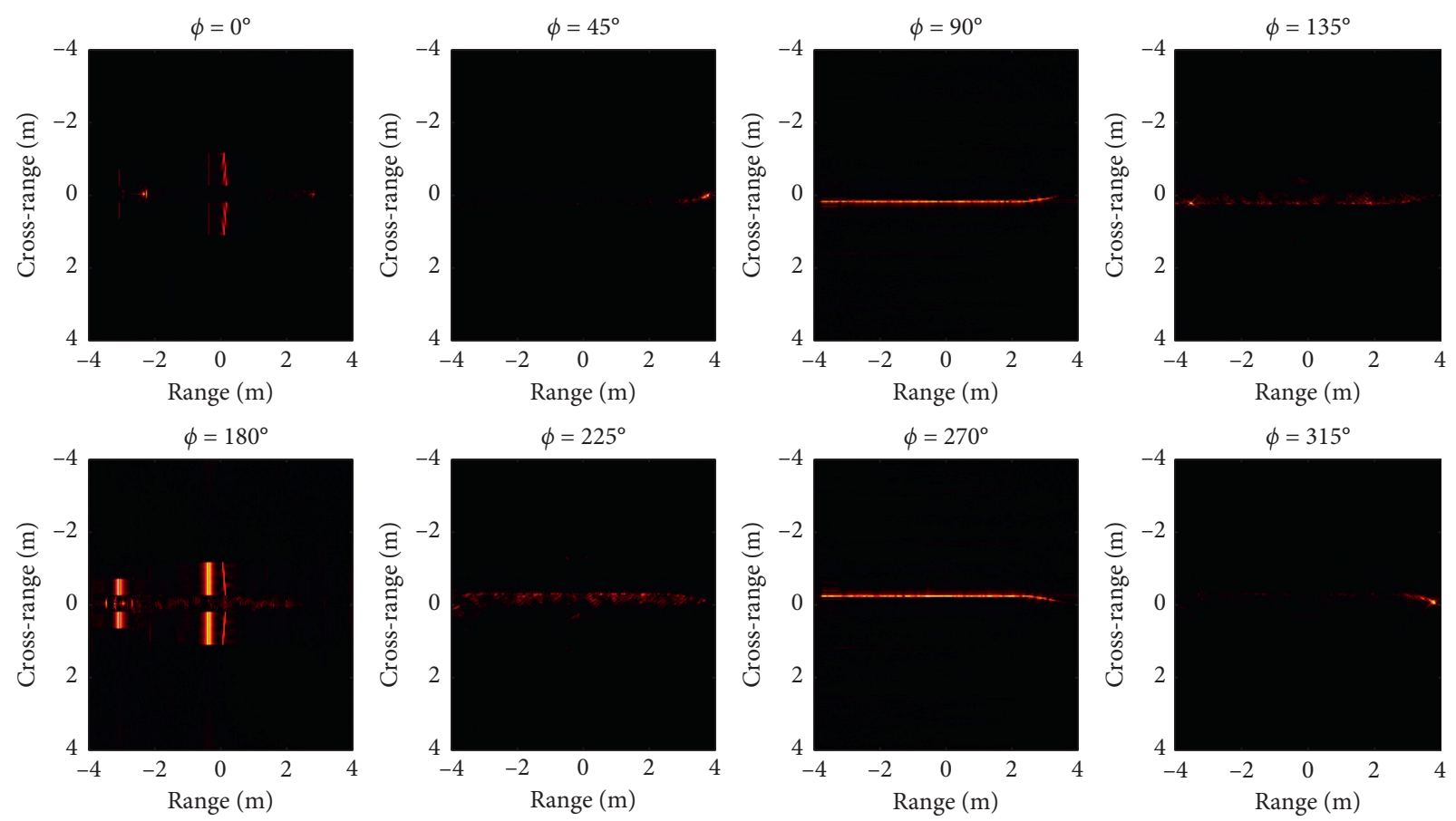

FIgURE 13: Subaperture images after rotation.

airplane model in the local coordinate system and global coordinate system, respectively. When the center azimuth is $0^{\circ}$, that is, the wave incidents from the nose of the airplane, strong backscattering from the airplane nose, and wings dominate the subimage. In the 3 th and 7 th subimages, the sides of the airplane are presented. 


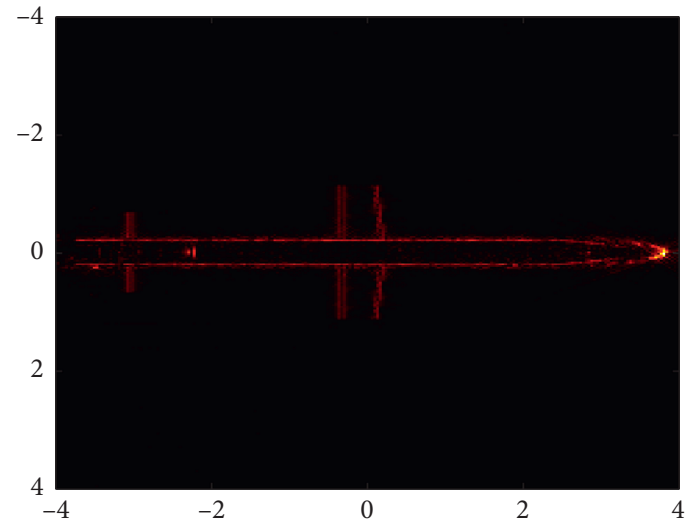

(a)

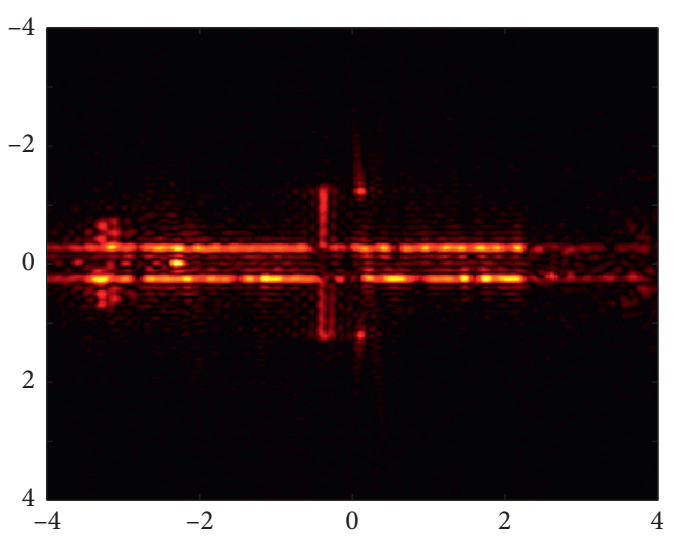

(b)

FIGURE 14: Final multiview ISAR image of the missile model. (a) Simulated by large-angle and wide-bandwidth data. (b) Simulated by smallangle and narrow-bandwidth data.
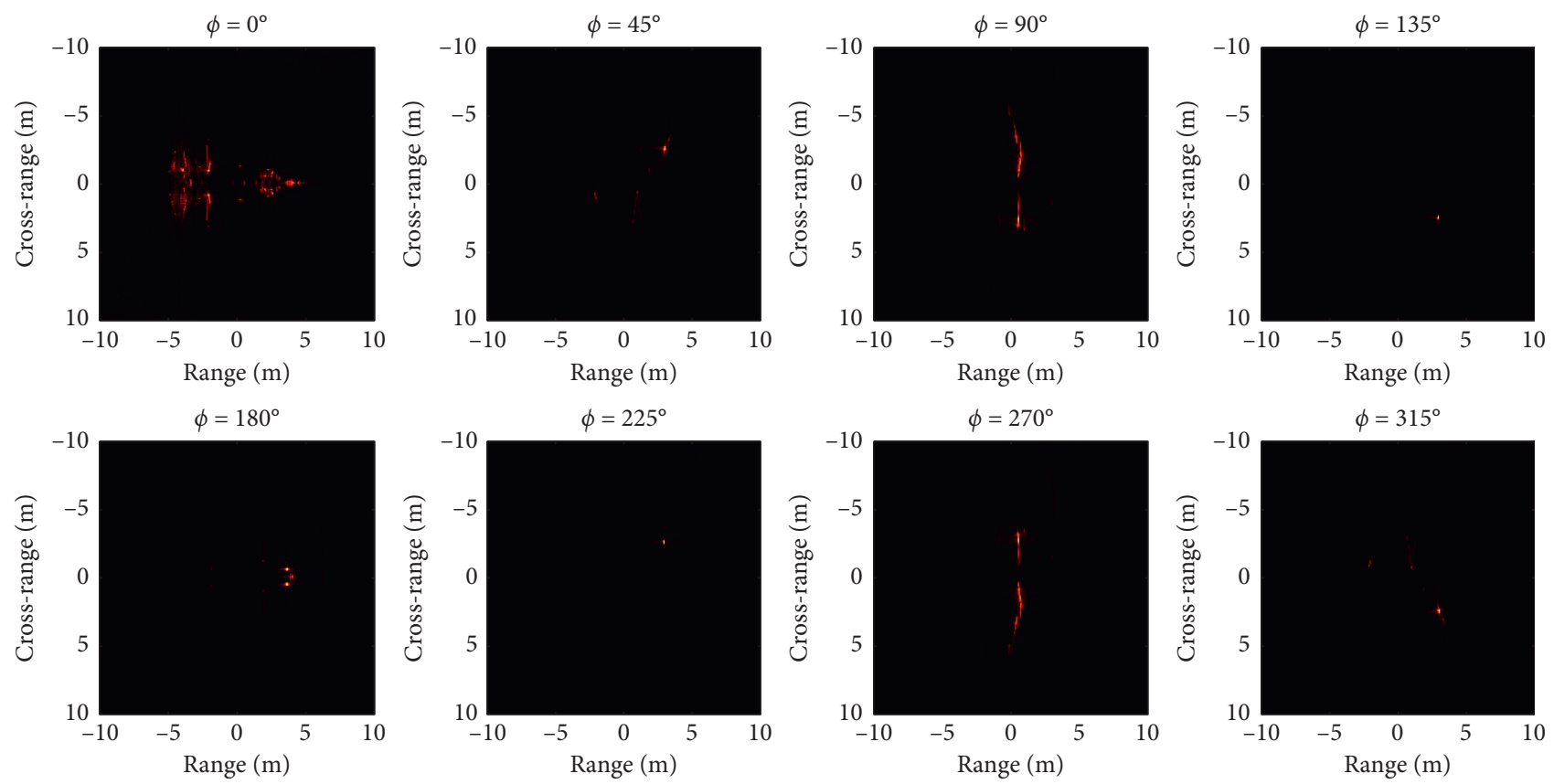

Figure 15: Subaperture images in different look-angles.

The other subimages show less target information because the backscattering intensity is relatively weak in those azimuths. Even so, these images are quite useful for SAR ATR to conduct recognition tests. The final multiview ISAR image of the airplane model is illustrated in Figure 17(a). It can be seen that the outline of the airplane model is clearly presented. Figure 17 (b) shows the multiview ISAR image simulated with small angle and narrow bandwidth. The angle range is $4.3^{\circ}$ and the frequency bandwidth is $750 \mathrm{MHz}$ for each subaperture. This ISAR image is blurrier compared with that in Figure 17(a). 

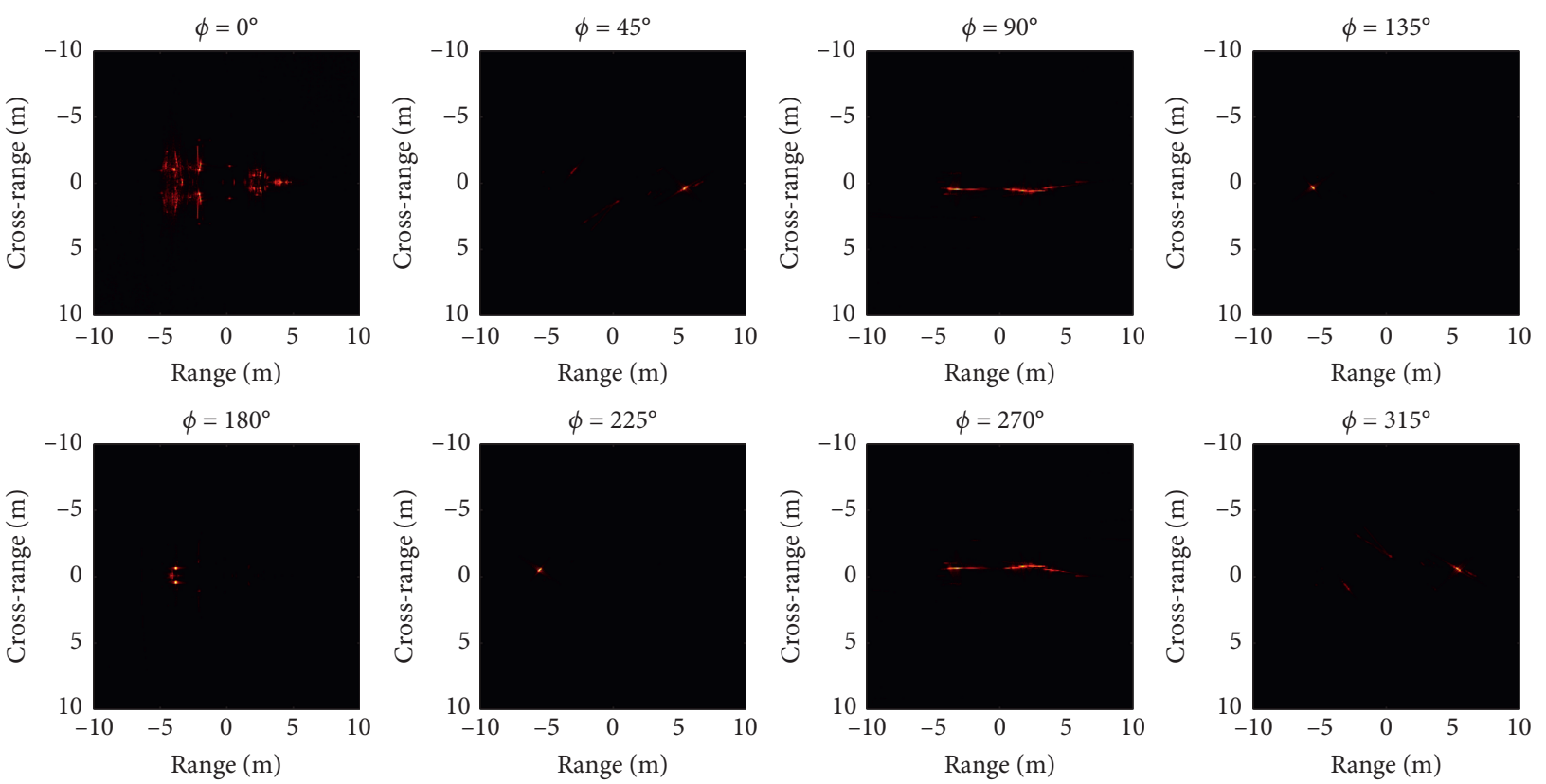

Figure 16: Subaperture images after rotation.

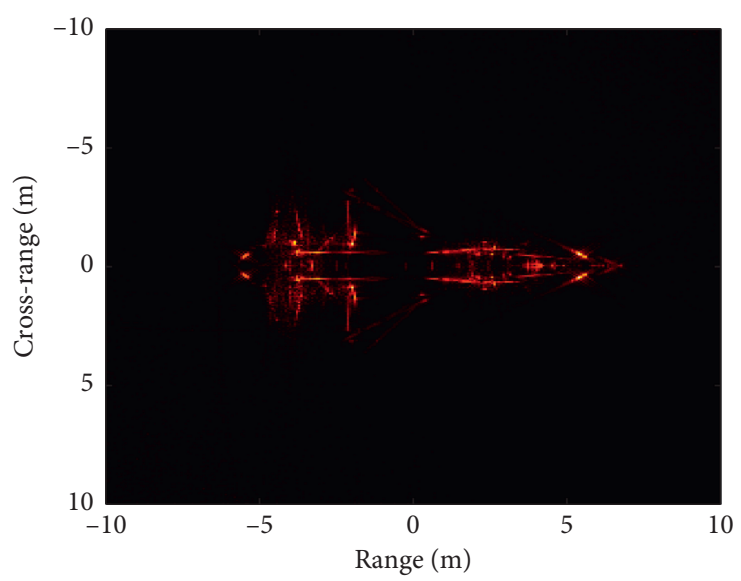

(a)

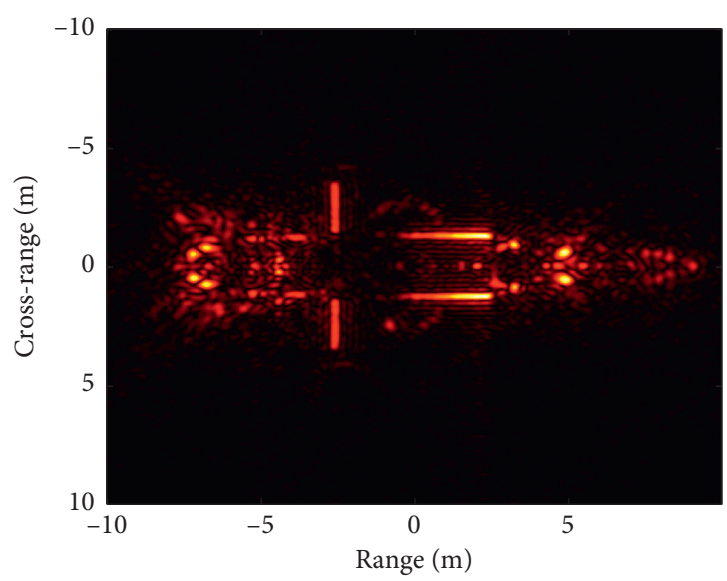

(b)

FIGURE 17: Final multiview ISAR image of the airplane model. (a) Simulated by large-angle and wide-bandwidth data. (b) Simulated by small-angle and narrow-bandwidth data.

\section{Conclusion}

In this paper, the multiview ISAR imaging method of electrically large objects with large-angle and wide-bandwidth data in each subaperture is proposed and validated. The scattering data are obtained by the improved SBR method using the bidirectional ray-tracing algorithm. The comparison of simulation results between the improved SBR method and MLFMM method in FEKO demonstrates that the improved method is able to calculate the backscattering of electrically large targets in high efficiency and accuracy. The final multiview ISAR image has good resolution to clearly outline the target model.

\section{Data Availability}

The scattering data used to support the findings of this study are available from the corresponding author upon request.

\section{Conflicts of Interest}

The authors declare that they have no conflicts of interest.

\section{Acknowledgments}

This work was supported in part by the National Natural Science Foundation of China under grant no. 61871457 and 
the Foundation for Innovative Research Groups of the National Natural Science Foundation of China under grant no. 61621005 .

\section{References}

[1] C. Özdemir, Inverse Synthetic Aperture Radar Imaging with MATLAB Algorithm, pp. 196-197, John Wiley \& Sons, Hoboken, NJ, USA, 2012.

[2] S. Kei and M. Iwamoto, "A two-dimensional bandwidth extrapolation technique for polarimetric synthetic aperture radar images," IEEE Transactions on Geoscience and Remote Sensing, vol. 45, no. 1, pp. 45-54, 2007.

[3] V. K. Nguyen and M. D. E. D. Turley, "Bandwidth extrapolation of LFM signals for narrowband radar systems," IEEE Transactions on Aerospace and Electronic Systems, vol. 51, no. 1, pp. 702-712, 2015.

[4] M. T. Alonso, P. López-Dekker, and J. J. Mallorquí, "A novel strategy for radar imaging based on compressive sensing," IEEE Transactions on Geoscience and Remote Sensing, vol. 48, no. 12, pp. 4285-4295, 2010.

[5] H. Yang, T. Li, Z. He, and Q. H. Liu, "Impulse borehole radar imaging based on compressive sensing," IEEE Geoscience and Remote Sensing Letters, vol. 12, no. 4, pp. 766-770, 2015.

[6] H. Zhai and C. Liang, "A simple iterative method for considering multibounce in PO region of MoM-PO," Microwave and Optical Technology Letters, vol. 40, no. 2, pp. 110-112, 2004.

[7] Z.-L. Liu, X. Wang, and C.-F. Wang, “A general MoM-PO hybrid framework for modelling complex antenna arrays mounted on extremely large platform (invited paper)," in Proceedings of the 2013 International Symposium on Antennas \& Propagation, pp. 48-51, Nanjing, China, October 2013.

[8] Z. Zubac, D. De Zutter, and D. Vande Ginste, "Scattering from two-dimensional objects of varying shape combining the multilevel fast multipole method (MLFMM) with the stochastic galerkin method (SGM)," IEEE Antennas and Wireless Propagation Letters, vol. 13, pp. 1275-1278, 2014.

[9] H. Ling, R.-C. Chou, and S.-W. Lee, "Shooting and bouncing rays: calculating the RCS of an arbitrarily shaped cavity," IEEE Transactions on Antennas and Propagation, vol. 37, no. 2, pp. 194-205, 1989.

[10] T.-Q. Fan, L.-X. Guo, and W. Liu, "A novel openGL-based MoM/SBR hybrid method for radiation pattern analysis of an antenna above an electrically large complicated platform," IEEE Transactions on Antennas and Propagation, vol. 64, no. 1, pp. 201-209, 2016.

[11] C.-L. Dong, L.-X. Guo, X. Meng, and Y. Wang, “An accelerated SBR for EM scattering from the electrically large complex objects," IEEE Antennas and Wireless Propagation Letters, vol. 17, no. 12, pp. 2294-2298, 2018.

[12] G. Guo and L. Guo, "SBR method for near-field scattering of an electrically large complex target illuminated by dipole sources," IEEE Access, vol. 6, pp. 78710-78718, 2018.

[13] P. Sundararajan and M. Y. Niamat, "FPGA implementation of the ray tracing algorithm used in the XPATCH software," in Proceedings of the 44th IEEE 2001 Midwest Symposium on Circuits and Systems. MWSCAS 2001, vol. 1, pp. 446-449, Dayton, OH, USA, August 2002.

[14] W. Huang and W. G. Wan, "A FPGA ray tracing scheme with memory optimization facility," in Proceedings of the IET International Communication Conference on Wireless Mobile and Computing (CCWMC 2011), pp. 329-334, Shanghai, China, 2011.
[15] S. Suk, T.-I. Seo, H.-S. Park, and H.-T. Kim, "Multiresolution grid algorithm in the SBR and its application to the RCS calculation," Microwave and Optical Technology Letters, vol. 29, no. 6, pp. 394-397, 2001.

[16] K.-S. Jin, T.-I. Suh, S.-H. Suk, B.-C. Kim, and H.-T. Kim, "Fast ray tracing using a space-division algorithm for RCS prediction," Journal of Electromagnetic Waves and Applications, vol. 20, no. 1, pp. 119-126, 2006.

[17] Y.-B. Tao, H. Lin, and H. J. Bao, "KD-tree based fast ray tracing for RCS prediction," Progress in Electromagnetics Research, vol. 81, pp. 329-341, 2008.

[18] D. Malmgren-Hansen, A. Kusk, J. Dall, A. A. Nielsen, R. Engholm, and H. Skriver, "Improving SAR automatic target recognition models with transfer learning from simulated data," IEEE Geoscience and Remote Sensing Letters, vol. 14, no. 9, pp. 1484-1488, 2017.

[19] B. Ding and G. Wen, "Target reconstruction based on 3-D scattering center model for robust SAR ATR," IEEE Transactions on Geoscience and Remote Sensing, vol. 56, no. 7, pp. 3772-3785, 2018.

[20] P. Van Dorp, M. P. G. Otten, and J. M. M. Verzeilberg, "Coherent multistatic ISAR imaging," in Proceedings of the IET International Conference on Radar Systems (Radar 2012), pp. 1-6, Glasgow, UK, October 2012.

[21] F. Salvetti, D. Staglianò, E. Giusti, and M. Martorella, "Multistatic 3D ISAR image reconstruction," in Proceedings of the 2015 IEEE Radar Conference, pp. 640-645, Arlington, VA, USA, May 2015.

[22] F. Salvetti, E. Giusti, D. Staglianò, and M. Martorella, "Incoherent fusion of 3D InISAR images using multi-temporal and multi-static data," in Proceedings of the 2016 IEEE Radar Conference, pp. 1-6, Philadelphia, PA, USA, May 2016.

[23] S. Brisken, "Multistatic ISAR-chances and challenges," in Proceedings of the 2014 International Radar Conference, Lille, France, October 2014.

[24] Y. Li and D. Zhu, "The geometric-distortion correction algorithm for circular-scanning SAR imaging," IEEE Geoscience and Remote Sensing Letters, vol. 7, no. 2, pp. 376-380, 2010.

[25] F. Salvetti, M. Martorella, E. Giusti, and D. Staglianò, "Multiview three-dimensional interferometric inverse synthetic aperture radar," IEEE Transactions on Aerospace and Electronic Systems, vol. 55, no. 2, pp. 718-733, 2019.

[26] D. Xu, G. Sun, and M. Xing, "Multi-view electromagnetic imagining," in Proceedings of the 2018 IEEE International Conference on Computational Electromagnetics (ICCEM), pp. 1-3, Chengdu, China, March 2018. 